\title{
Availability and Utilization of Health Education Methods for Clients in Primary Health Care Centers in Makkah, Saudi Arabia
}

\author{
Mohammed Mosleh Alameer ${ }^{1}$, Khalid Iyash Alshareef ${ }^{1}$, Hussein Hamied alkaf ${ }^{1}$, \\ Fahad Brakah Alharbi ${ }^{1}$, Abdullah Saeed Abdullah ${ }^{1}$, Anas Seraj Dablool ${ }^{2}$, \\ Saad Saeed Alghamdi ${ }^{2}$, Asim Lutfi Jilani ${ }^{1}$. Abdullah Jameel Halawany ${ }^{3}$. \\ Ministry of Health, Saudi arabia ${ }^{1}$. Umm Alqura University, Saudi Arabia ${ }^{2}$. University of Central Folorida, \\ $U S A^{3}$
}

\begin{abstract}
The aim of this study is to know the availability and utilization of health education methods for Clients in Primary Health Care Centers in Makkah, Saudi Arabia according to the opinion of clients. A total of 200 clients over 18 years old in about 18 health care centers were interviewed to obtain their opinions in health care programs that delivered by health care centers. According to the findings, T.V was available for most $166(83 \%)$ of clients in primary health care centers, followed by internet $113(56 \%)$ and radio $86(43 \%)$, while exhibitions were available for only $7 \%$ of clients. Also it has been found that highest percentage (73\%) of study group follow health education activities in T.V, radio 50\%, internet $42.5 \%$ and exhibitions $16.5 \%$. Most of primary health clients $144(72 \%)$ were not satisfied from available health education activities. The study concluded that T.V, Internet and radio were the top source and satisfied method of health education for public.
\end{abstract}

Keywords: health, education, internet, patients, Makkah

\section{Introduction}

Health services in Saudi Arabia have increased and improved significantly during recent decades [1]. Health education is essential one of these delivered health education to improve health status of people. Health education is defined as any combination of learning experiences designed to help individuals and communities improve their health, by increasing their knowledge or influencing their attitudes [2]. There are many methods that could be used in health education directed to individuals and groups.

Health education is considered as a key to achieving better outcomes of health care. Satisfaction of patients regarding health education services is associated with improvements in the quality of health care and improved health outcomes [3].

Anwar [4] reported that the health education programmes in Saudi Arabia are insufficient to meet the high demand in both rural and urban areas, in addition to that the quality of these programmes (particularly in managed by primary health centres) suffers from a lack of clearly communicated health goals, theory-based interventions, communication behavioural-related outcomes and comprehensiveness.

\section{Materials And Methods}

The study was conducted in primary health care centers in Makkah Almokarramah, Saudi Arabia, which the Holy town that receives million of people from world annually to do Hajj and Omrah. A total of 200 people over 18 years old who were attending (clients) in 18 health care centers were interviewed to obtain their opinions availability, utilization and satisfaction of health education methods used in primary health care centers.

\section{Results}

In the present study table 1 shows that T.V was available as health education methods for $83 \%$ of clients in primary health care centers, internet (56\%), roads signs (20\%), SMS (39\%), prints (33\%), field visits $(10.5 \%)$, radio $(86 \%)$, lectures and seminars $(17.5 \%)$, and exhibitions $(7 \%)$. Table 2 illustrates that $73 \%$ of clients (patients) followed T.V as a health education methods internet $(42.5 \%)$, roads signs $(38.5 \%)$, SMS (37.5\%), prints (36\%), field visits (26.5\%), radio (25\%), lectures and seminars (25\%), and exhibitions (16.5\%). Most of primary health clients $144(72 \%)$ were not satisfied from available health education activities. 
Table (1): Availability of health education methods according to the opinion of clients in health care centers in the Makkah Elmokarramah, Saudi Arabia

\begin{tabular}{|l|c|c|c|c|}
\hline \multirow{3}{*}{ Educational means } & Available & Not available & \multirow{2}{*}{ Medium } & Standard deviation \\
\cline { 2 - 3 } & No $(\%)$ & No $(\%)$ & 1.8300 & .37658 \\
\hline T.V & $166(83 \%)$ & $34(17 \%)$ & 1.5750 & .52512 \\
\hline Road signs & $113(56.5 \%)$ & $86(43.5 \%)$ & 1.2000 & .40100 \\
\hline SMS & $40(20 \%)$ & $160(80 \%)$ & 1.3900 & .48897 \\
\hline Prints & $78(39 \%)$ & $122(61 \%)$ & 1.3300 & .47139 \\
\hline Field visits & $66(33 \%$ & $134(67 \%)$ & 1.1050 & .30732 \\
\hline Radio & $21(10.5 \%)$ & $179(89.5 \%)$ & 1.4300 & .49632 \\
\hline Lectures\& Seminars & $86(43 \%)$ & $114(57 \%)$ & 1.1750 & .38092 \\
\hline exhibitions & $35(17.5 \%)$ & $165(82.5 \%)$ & 1.0700 & 25579 \\
\hline
\end{tabular}

Table (2): Utilization of health education methods by clients in health care centers in Makkah Elmokarramah, Saudi Arabia

\begin{tabular}{|l|c|c|c|c|}
\hline \multirow{2}{*}{ Educational means } & followed & Not followed & \multirow{2}{*}{ Medium } & Standard deviation \\
\cline { 2 - 3 } & No $(\%)$ & No $(\%)$ & 1.7300 & .44507 \\
\hline T.V & $146(73 \%)$ & $54(27 \%)$ & 1.4250 & .49558 \\
\hline Internet & $85(42.5 \%)$ & $115(57.5 \%)$ & 1.3850 & .48782 \\
\hline Road signs & $77(38.5 \%)$ & $123(61.5 \%)$ & 1.3750 & .48534 \\
\hline Prins & $75(37.5 \%)$ & $125(62.5 \%)$ & 1.3600 & .48120 \\
\hline Field visits & $72(36 \%)$ & $128(64 \%)$ & 1.2650 & .44244 \\
\hline Radio & $53(26.5 \%)$ & $147(73.5 \%)$ & 1.2500 & .43410 \\
\hline Lectures\& Seminars & $50(25 \%)$ & $150(75 \%)$ & 1.2500 & .43410 \\
\hline exhibitions & $50(25 \%)$ & $150(75 \%)$ & 1.1650 & .37211 \\
\hline
\end{tabular}

Table (3): Satisfaction of clients as regard to health education delivered by available methods in health care centers in the Makkah Elmokarramah, Saudi Arabia

\begin{tabular}{|l|c|c|}
\hline Satisfaction & No & $\%$ \\
\hline Satisfied & 56 & $28 \%$ \\
\hline Not satisfied & 144 & $72 \%$ \\
\hline Total & 200 & 100 \\
\hline
\end{tabular}

\section{Discussion}

It is of paramount importance to deliver health education in primary health care setting, because patients need to know their conditions and how they deal with to achieve recovery and to prevent their families and community members. In this study, several methods of health education were used in primary health care in Makkah to deliver health messages to patients as one of primary health care. T.V is still the frequent methods to get most of health information and alert; it might be attributed to the many reasons e.g. availability, mass media and needs no education and skills to follow. Television has proved to be a profound means of delivery of a lot of information including health one. Ezekiel and Peter, [5] found that radio was mostly used to access information followed by mobile phone, television, newspaper, social network, satellite and cable television followed by the internet. The second available and used method in this study was internet. In a study conducted Among French Young Adults, it has been found that The Internet is a useful tool to spread health information and prevention campaigns, especially to target young adults [6]. Also Jay and John [7] found that the Internet is starting to revolutionize health education research and practice. Findings obtained from Saudi Arabia, about $44.8 \%$ of the patients have received printed educational materials, group teaching, education exhibition and advices given in the waiting area $(24 \%, 19.5 \%, 12.8 \%$ and $20.2 \%)$ respectively [3].

\section{Conclusion}

There are many different methods that could be used as health education tools for public; however health education activities delivered by these methods were not satisfied from study group point of view.

\section{Acknowledgment}

Thanks to administrations, employees and study group in all participated health centers in Makkah Almokarramah for their assistance and participation.

\section{References}

[1] M. Almalki, G. Fitzgerald and M. Clark, Health care system in Saudi Arabia: an overview. Eastern MediterraneanHealth Journal 17(10), 2011, $784-793$.

[2] Fasoranti, Afolabi Joseph and Adeyeye, Mayowa Festus., Health Education as a Tool for Effective Primary Health Care Services in Nigeria. Journal of Emerging Trends in Educational Research and Policy Studies (JETERAPS) 6(7), 2015, $225-228$. 
[3] Nadia Asiri1, Amen A Ahmed Bawazir and Hoda Jradi. (2013). Patients' Satisfaction with Health Education Services at Primary Health Care Centers in Riyadh, KSA. J Community Med Health Educ 4(1): 1000268.

[4] Anwar Al-Hashim. (2016). Health Education in Saudi Arabia Historical overview. Sultan Qaboos University Med J,16(3): e286292.

[5] Familusi, Ezekiel Babatope and Owoeye, Peter Olufemi. ((2014).) "An Assessment of the Use of Radio and other Means of Information Dissemination by the Residents of Ado- Ekiti, Ekiti-State, Nigeria." Library Philosophy and Practice (e-journal). Paper 1088.

[6] François Beck, Jean-Baptiste Richard, Viet Nguyen-Thanh, Ilaria Montagni, Isabelle Parizot, and Emilie Renahy. (2014). Use of the Internet as a Health Information Resource Among French Young Adults: Results From a Nationally Representative Survey. J Med Internet Res, 16(5): e128.

[7] Jay M. Bernhardt, John Hubley. (2001). Health education and the Internet: the beginning of a revolution. Health Educ Res, 16 (6): 643-645. 